\title{
The Fundamental Theorem of Asset Pricing with either Frictionless or Frictional Security Markets
}

\author{
Helen H. Huang ${ }^{1}$, Shunming Zhang ${ }^{2 *}$ \\ ${ }^{1}$ Faculty of Business Administration, The University of Regina, Regina, Canada \\ ${ }^{2}$ School of Finance, Renmin University of China, Beijing, China \\ Email: helen.huang@uregina.ca, szhang@ruc.edu.cn, shunning.zhang@gmail.com
}

Received November 21, 2013; revised January 6, 2014; accepted January 22, 2014

Copyright (C) 2014 Helen H. Huang, Shunming Zhang. This is an open access article distributed under the Creative Commons Attribution License, which permits unrestricted use, distribution, and reproduction in any medium, provided the original work is properly cited. In accordance of the Creative Commons Attribution License all Copyrights (C) 2014 are reserved for SCIRP and the owner of the intellectual property Helen H. Huang, Shunming Zhang. All Copyright (C 2014 are guarded by law and by SCIRP as a guardian.

\section{ABSTRACT}

This paper studies asset pricing in arbitrage-free financial markets in general state space (both for frictionless market and for market with transaction cost). The mathematical formulation is based on a locally convex topological space for weakly arbitrage-free securities' structure and a separable Banach space for strictly arbitragefree securities' structure. We establish, for these two types of spaces, the weakly arbitrage-free pricing theorem and the strictly arbitrage-free pricing theorem, respectively.

\section{KEYWORDS}

The First Fundamental Valuation Theorems; Transaction Costs; Weak Arbitrage-Freeness; Strict Arbitrage-Freeness; Arbitrage-Free Pricing Theory; Locally Convex Topological Space; Separable Banach Space

\section{Introduction}

Arbitrage-free asset pricing theory is of fundamental importance in neo-classical finance. In this paper, we consider it in a setting of general state spaces, in particular, locally convex topological space for weakly arbitrage-free security markets, and separable Banach space for strictly arbitrage-free security markets. We deal with both frictionless and frictional security markets.

In the study of mathematical economics, arbitrage-free conditions have always been an important first step toward the general equilibrium theorem with incomplete asset markets (Duffie and Shafer [1,2]; Geanakoplos [3]; Geanakoplos and Shafer [4]; Hirsch, Magill and Mas-Colell [5]; Husseini, Lasry and Magill [6]; and Magill and Shafer [7]). Since the 1980s, for finite period economies, no-arbitrage pricing theory has been applied by various authors to prove the existence of general equilibrium for stochastic economies with incomplete financial markets (Duffie [8-10]; Florenzano and Gourdel [11]; Magill and Shafer [7]; Werner [12,13]; and Zhang [14]). In those works, the finite number of possible states of nature and the finite-dimensional commodity space are usually assumed in order for the proofs to be carried out for the general equilibrium model with incomplete financial markets. Our model considers a general state space which may have an infinite number of states.

Many mathematical economists usually apply Stiemke's Lemma, a strict version of Farkas-Minkowski's Lemma, to study the asset pricing theory with no-arbitrage conditions, for example, discrete-time models of dynamic asset pricing theory (Duffie $[9,10]$ ) and the theory of economic equilibrium with incomplete asset markets (Geanakoplos [3]; Geanakoplos and Shafer [4]; Hirsch, Magill and Mas-Colell [5]; Husseini, Lasry and Magill [6]; and Magill and Shafer [7]) where the commodity space is of finite dimension. Farkas-Minkowski's Lemma and Stiemke's Lemma are in essence the mathematical counter part of the asset pricing theory with no-arbitrage conditions. In this paper, we obtain extensions (to the general state space in our discussion) of

\footnotetext{
${ }^{*}$ Corresponding author.
} 
Farkas-Minkowski’s Lemma and Stiemke's Lemma by applying Clark’s separating hyperplane theorems (Clark $[15,16])$, and thus establish our main results.

Harrison and Kreps [17] initiated the study of martingales and arbitrage in multiperiod security markets. They first introduced general theory of arbitrage in a two-period economy with uncertainty, and then extended it to the models of multiperiod security markets and the models of continuous-time securities markets. Kreps [18] studied arbitrage and equilibrium in economies with infinitely many commodities and presented an abstract analysis of "arbitrage" in economies that have infinite dimensional commodity space. Harrison and Pliska [19] studied martingales and stochastic integrals in the theory of continuous trading. Dalang, Morton and Willinger [20] studied equivalent martingale measures and no-arbitrage in stochastic securities market models. Back and Pliska [21] studied the fundamental theorem of asset pricing with an infinite state space and showed some equivalent relations on arbitrage. Jacod and Sgiryaev [22] studied local martingales and the fundamental asset pricing theorems in the discrete-time case. These papers studied fundamental theorems of asset pricing in multiperiod financial models with the help of techniques from stochastic analysis. Our work is based on separating hyperplane theorems and does not rely on assumptions made for stochastic analysis to be able to carry out in the above models.

Friction in markets has attracted attention of several works in this field recently. Chen [23] examined the incentives and economic roles of financial innovation and at the same time studied the effectiveness of the replication-based arbitrage valuation approach in frictional economies (the friction means holding constraints). Jouini and Kallal [24] derived the implications of the absence of arbitrage in securities markets models where traded securities are subject to short-sales constraints and where the borrowing and lending rates differ, and showed that a securities price system is arbitrage free if and only if there exists a numeraire and an equivalent probability measure for which the normalized (by the numeraire) price processes of traded securities are supermartingales. Jouini and Kallal [25] derived the implications from the absence of arbitrage in dynamic securities markets with bid-ask spreads. The absence of arbitrage is equivalent to the existence of at least an equivalent probability measure that transforms some process between the bid and the ask price processes of traded securities into a martingale. Pham and Touzi [26] addressed the problem of characterization of no arbitrage (strictly arbitrage-free) in the presence of friction in a discrete-time financial model, and extended the fundamental theorem of asset pricing under a non-degeneracy assumption. The friction is described by the transaction cost rates for purchasing and selling the securities. We follow the model for transaction costs from Pham and Touzi [26], and then extend the first fundamental valuation theorems of asset pricing from frictionless security markets to frictional security markets, for general state space. Again, their stochastic analysis method requires stronger assumptions than ours. In addition, their arbitrage-free conditions are slightly different from ours based on that of Duffie [9].

Section 2 presents our model without transaction costs, we define the weakly arbitrage-free security markets and strictly arbitrage-free security markets, following Duffie [9]. In the next two sections, we establish the first fundamental valuation theorem of asset pricing (a necessary and sufficient condition for arbitrage-freeness) with weakly arbitrage-free security markets in Section 3 and strictly arbitrage-free security markets in Section 4. In the rest of the paper we extend our work to markets with transaction costs, following the model of Pham and Touzi [26]. The transaction cost model is presented in Section 5, and the corresponding first fundamental valuation theorems of asset pricing are done in Sections 6 and 7. Section 8 concludes our article with some remarks.

\section{Frictionless Security Markets}

We consider a two-period model (dates 0 and 1 ) with uncertainty over the states of nature in the date 1 . The unknown nature of the future is represented by a general set $\Omega$ of possible states of nature, one of which will be revealed as true. Here we make no assumption about the probability of these states. The $J$ securities are given by a return "matrix" $V=\left(V^{1}, \cdots, V^{J}\right)$, where $V^{j}$ denotes the number of units of account paid by security $j=1, \cdots, J$. Let $q \in \mathcal{R}^{J}$ denote the vector of prices of $J$ securities. A portfolio $\theta \in \mathcal{R}^{J}$ has market value $q^{\mathrm{T}} \theta$ and payoff $V \theta$.

Let $T$ be a topological space consisting of processes in $\mathcal{R}^{\Omega}, T_{+}$is the positive cone of $T$. Let $T^{*}$ be the dual space composed of the continuous linear functionals on $T, T_{+}^{*}$ the positive cone of the space $T^{*}$ (the space of all positive continuous linear functionals on $T$ ) and $T_{++}^{*}$ the interior of the cone $T_{+}^{*}$ (the space of all strictly positive continuous linear functionals on $T$ ): $T_{++}^{*} \subseteq T_{+}^{*}$. 
In this paper, we assume $V^{j} \in T$ for $j=1, \cdots, J$. Then $V \theta=\sum_{j=1}^{J} V^{j} \theta^{j} \in T$. Our proof must adopt the following notation $\langle V\rangle=\left\{V \theta \in T \mid \theta \in \mathcal{R}^{J}\right\}$ and $\langle V\rangle_{+}=\langle V\rangle \cap T_{+}$.

[Definition 1] The frictionless market $(q, V)$ is weakly arbitrage-free if any portfolio $\theta \in \mathcal{R}^{J}$ of securities has a positive market value $q^{\mathrm{T}} \theta \geq 0$ whenever it has a positive payoff $V \theta \in T_{+}$.

[Definition 2] The frictionless market $(q, V)$ is strictly arbitrage-free if (1) any portfolio $\theta \in \mathcal{R}^{J}$ of securities has a strictly positive market value $q^{\mathrm{T}} \theta>0$ whenever it has a positive non-zero payoff $V \theta \in T_{+} \backslash\{0\}$; and (2) any portfolio $\theta \in \mathcal{R}^{J}$ of securities has a zero market value $q^{\mathrm{T}} \theta=0$ whenever it has a zero payoff $V \theta=0$.

Definition 2 implies Definition 1 obviously. We follow the definition for arbitrage opportunity as provided by Duffie [9,10]. An arbitrage is a portfolio $\theta \in \mathcal{R}^{J}$ with either (1) $q^{\mathrm{T}} \theta \leq 0$ and $V \theta \in T_{+} \backslash\{0\}$, or (2) $q^{\mathrm{T}} \theta<0$ and $V \theta \in T_{+}$. That is to say, the frictionless market $(q, V)$ admits an arbitrage opportunity if there exists an portfolio $\theta \in \mathcal{R}^{J}$ of securities such that either (1) $q^{\mathrm{T}} \theta \leq 0$ and $V \theta \in T_{+} \backslash\{0\}$, or (2) $q^{\mathrm{T}} \theta<0$ and $V \theta \in T_{+}$. Consequently, we can define the strictly arbitrage-free (no arbitrage) frictionless market $(q, V)$ as follows.

[Definition 2'] The frictionless market $(q, V)$ is strictly arbitrage-free if (1) any portfolio $\theta \in \mathcal{R}^{J}$ of securities has a strictly positive market value $q^{\mathrm{T}} \theta>0$ whenever it has a positive non-zero payoff $V \theta \in T_{+} \backslash\{0\}$; and (2) any portfolio $\theta \in \mathcal{R}^{J}$ of securities has a positive market value $q^{\mathrm{T}} \theta \geq 0$ whenever it has a positive payoff $V \theta \in T_{+}$.

[Lemma 1] Definitions 2 and 2' are equivalent.

An arbitrage is therefore, in effect, a portfolio offering "something for nothing". Not surprisingly, an arbitrage is naturally ruled out in reality. And this fact gives a characterization of security prices as follows: A valuation functional is a functional $v \in T_{+}^{*}$ for the weakly arbitrage-free frictionless market $(q, V)$ with consistency $q^{\mathrm{T}}=v V$; and a functional $v \in T_{++}^{*}$ for the strictly arbitrage-free frictionless market $(q, V)$ with consistency $q^{\mathrm{T}}=v V$, where $v V=\left(v V^{1}, \cdots, v V^{J}\right)$. The valuation functional is called to be a positive linear consistent valuation operator for the weakly arbitrage-free frictionless market, a strictly positive linear consistent valuation operator for the strictly arbitrage-free frictionless market, respectively.

The idea of arbitrage and the absence of arbitrage opportunities is fundamental in finance. The strict arbitrage-freeness is important in the study of general equilibrium theory with incomplete asset markets (Husseini, Lasry and Magill [6]; Werner [13]; and Magill and Shafer [7]). Theorem 2 to be presented in the following is an important step in the study of equilibrium for economies with general state spaces considered in our work. The principal mathematical tool applied here is the Separating Hyperplane Theorems of Clark [15] and [16].

[Fact 1] (Clark [16]) Suppose $M$ and $N$ are non-empty disjoint convex cones in a locally convex topological vector space $E$. Then there exists a non-zero continuous linear functional $f: E \rightarrow R$ separating $N$ from $M: f(n) \geq 0$ for all $n \in N$ and $f(m) \leq 0$ for all $m \in M$ if and only if $\overline{M-N} \neq E$. Moreover, if $\overline{M-N} \neq E$, then for any $e \notin \overline{M-N}$ we may select $f$ so that $f(e)>0$.

[Fact 2] (Clark [15]) Suppose $M$ and $N$ are non-empty convex cones (with vertices at the origin) in a separating Banach space $E$. Then there exists a non-zero continuous linear functional $f: E \rightarrow \mathcal{R}$ strictly separating $N$ from $M: \quad f(n)>0$ for all $n \in N$ and $f(m) \leq 0$ for all $m \in M$ if and only if $N \cap \overline{M-N}=\varnothing$.

Fact 1 and 2 will be used to prove Theorems 1 and 2 in Sections 3 and 4, Theorems 3 and 4 in Sections 6 and 7, respectively. We assume $E=\mathcal{R} \times T$, which is a topological space, then $E_{+}=\mathcal{R}_{+} \times T_{+}$is the positive cone of $E$, which is a positive closed convex cone of $E$ with its vertex at the origin. The marketed subspace

$$
M=\left\{\left(-q^{\mathrm{T}} \theta, V \theta\right) \in E \mid \theta \in \mathcal{R}^{J}\right\}
$$

is a linear subspace of the space $E$.

\section{Weakly Arbitrage-Free Security Valuation Theorem with Frictionless Security Markets}

In this section, we assume that $T$ is a locally convex topological space.

[Proposition 1] The frictionless market $(q, V)$ is weakly arbitrage-free if and only if $M \cap E_{+} \subseteq\{0\} \times\langle V\rangle_{+}$. 
Proof. $(r, t) \in M \cap E_{+}$implies that there exists $\theta \in \mathcal{R}^{J}$ such that $r=-q^{\mathrm{T}} \theta \geq 0$ and $t=V \theta \in T_{+}$. On the other hand, $V \theta \in T_{+}$implies that $q^{\mathrm{T}} \theta \geq 0$ from Definition 1 , then $r=-q^{\mathrm{T}} \theta \leq 0$. Thus $r=-q^{\mathrm{T}} \theta=0$ and $t \in\langle V\rangle_{+}$, that is, $M \cap E_{+} \subseteq\{0\} \times\langle V\rangle_{+}$.

Conversely, if there exists $\theta \in \mathcal{R}^{J}$ such that $V \theta \in T_{+}$and $q^{\mathrm{T}} \theta<0$, then $-q^{\mathrm{T}} \theta>0$. Thus

$$
\left(-q^{\mathrm{T}} \theta, V \theta\right) \in M \cap E_{+} \text {and }\left(-q^{\mathrm{T}} \theta, V \theta\right) \notin\{0\} \times\langle V\rangle_{+} .
$$

This is a contradiction! Therefore the frictionless market $(q, V)$ is weakly arbitrage-free. Q.E.D.

[Theorem 1] The frictionless market $(q, V)$ is weakly arbitrage-free if and only if there exists a positive functional $v \in T_{+}^{*}$ satisfying $q^{\mathrm{T}}=v V$.

Proof. Both $M$ and $E_{+}$are closed and convex cones of $E$ with their vertices at the origin.

The frictionless market $(q, V)$ is weakly arbitrage-free if and only if $M \cap E_{+} \subseteq\{0\} \times\langle V\rangle_{+}$. Note that $M \cap E_{+}$is a convex set. Let $N=E_{+} \backslash M=E_{+} \backslash\left(M \cap E_{+}\right)$, then $N$ is a (non-empty) convex set. Both $M$ and $N$ are non-empty disjoint convex cones $M \cap N=M \cap\left(E_{+} \backslash M\right)=\varnothing .(1,0) \notin \overline{M-N}$ implies $\overline{M-N} \neq E$. Clark [16] stated that there exists a non-zero continuous linear functional $f: E \rightarrow \mathcal{R}$ separating $N$ from $M$, that is, $f(n) \geq 0$ for all $n \in N$ and $f(m) \leq 0$ for all $m \in M$. Moreover, $f(1,0)>0$.

$f: E \rightarrow \mathcal{R}$ is a positive continuous linear functional on $E$. In fact, for any $e \in E_{+}$and natural number $k=1,2, \cdots, e+\left(\frac{1}{k}, 0\right) \in N$ and $\lim _{k \rightarrow \infty} e+\left(\frac{1}{k}, 0\right)=e$. Therefore, $f\left(e+\left(\frac{1}{k}, 0\right)\right) \geq 0$. Thus

$$
f(e)=\lim _{k \rightarrow \infty} f\left(e+\left(\frac{1}{k}, 0\right)\right) \geq 0 .
$$

That is, $f$ is a positive continuous linear functional on $E$.

Thus $f$ is represented by some $\alpha \in \mathcal{R}_{++}$and $\beta \in T_{+}^{*}$ by $f(r, t)=\alpha r+\beta t$ for any $(r, t) \in E$. In fact, $f$ is represented by some $\alpha \in \mathcal{R}$ and $\beta \in T^{*}$ by $f(r, t)=\alpha r+\beta t$ for any $(r, t) \in E$. The fact that $(1,0) \notin \overline{M-N}$ implies $\alpha=f(1,0)>0$. If $\beta \notin T_{+}^{*}$, then there exists $t_{0} \in T_{+}$such that $\beta t_{0}<0$. Take $r_{0}=\frac{1}{2 \alpha}\left(-\beta t_{0}\right)>0$, then $\left(r_{0}, t_{0}\right) \in N$ and $f\left(r_{0}, t_{0}\right)=\alpha r_{0}+\beta t_{0}=\frac{1}{2} \beta r_{0}<0$. The contradiction means $\beta \in T_{+}^{*}$.

Since $M$ is a linear space, $f(m)=0$ for all $m \in M$, that is, $-\alpha q^{\mathrm{T}} \theta+\beta V \theta=0$ for all $\theta \in \mathcal{R}^{J}$. Then $q^{\mathrm{T}}=\left(\frac{1}{\alpha} \beta\right) V$. And therefore, the vector $\frac{1}{\alpha} \beta \in T_{+}^{*}$ is that we want.

The converse is obvious. Q.E.D.

[Remark 1] If $\Omega$ is a finite set, then $T=\mathcal{R}^{\Omega}$ is an Euclidean space, Definition 1 is the usual concept of weak arbitrage-freeness. Theorem 1 is the well-known Farkas-Minkowski’s Lemma (Duffie [9]; Farkas [27] and Franklin [28]).

[Remark 2] Theorem 1 (The Extension of Farkas-Minkowski’s Lemma) means that the present value of the securities prices at date 0 is the value of their returns at date 1 .

\section{Strictly Arbitrage-Free Security Valuation Theorem with Frictionless Security Markets}

In this section, we assume that $T$ is a separable Banach space. We prove Proposition 2 and Theorem 2 by using Definition 2 of the strictly arbitrage-free frictionless market $(q, V)$.

[Proposition 2] The frictionless market $(q, V)$ is strictly arbitrage-free if and only if $M$ and $E_{+}$intersect precisely at $(0,0)$, that is, $M \cap E_{+}=\{(0,0)\}$.

Proof. $(r, t) \in M \cap E_{+}$implies that there exists $\theta \in \mathcal{R}^{J}$ such that $r=-q^{\mathrm{T}} \theta \geq 0$ and $t=V \theta \in T_{+}$. If $V \theta \in T_{+} \backslash\{0\}$, then $q^{\mathrm{T}} \theta>0$ from Definition 2 (1), $r=-q^{\mathrm{T}} \theta \notin R_{+}$, a contradiction! If $V \theta=0 \in T_{+}$, then $q^{\mathrm{T}} \theta=0$ from Definition 2 (2), $r=-q^{\mathrm{T}} \theta=0$ and $t=V \theta=0 \in T_{+}$. Thus $M \cap E_{+} \subseteq\{(0,0)\}$. And hence, $M \cap E_{+}=\{(0,0)\}$.

For sufficiency, we must consider two cases: (1) If there exists $\theta \in R^{J}$ such that $V \theta \in T_{+} \backslash\{0\}$ and $q^{\mathrm{T}} \theta \leq 0$, then $-q^{\mathrm{T}} \theta \geq 0$ and $(0,0) \neq\left(-q^{\mathrm{T}} \theta, V \theta\right) \in M \cap E_{+}$, a contradiction. Therefore, any portfolio $\theta \in \mathcal{R}^{J}$ of securities has a positive non-zero market value $q^{\mathrm{T}} \theta>0$ whenever it has a positive payoff $V \theta \in T_{+} \backslash\{0\}$. (2) 
If there exists $\theta \in \mathcal{R}^{J}$ such that $V \theta=0 \in T_{+}$and $q^{\mathrm{T}} \theta \neq 0$, we discuss the problem for the following two settings. (2.1) When $q^{\mathrm{T}} \theta<0$, then $-q^{\mathrm{T}} \theta>0$, thus $(0,0) \neq\left(-q^{\mathrm{T}} \theta, V \theta\right) \in M \cap E_{+}$. This is a contradiction! (2.2) When $q^{\mathrm{T}} \theta>0$, then $-q^{\mathrm{T}}(-\theta)>0$ and $V(-\theta)=-V \theta=0$, thus $(0,0) \neq\left(-q^{\mathrm{T}}(-\theta), V(-\theta)\right) \in M \cap E_{+}$. This is a contradiction! The two contradictions mean that the frictionless market $(q, V)$ is strictly arbitrage-free. Q.E.D.

[Theorem 2] The frictionless market $(q, V)$ is strictly arbitrage-free if and only if there exists a strictly positive functional $v \in T_{++}^{*}$ satisfying $q^{\mathrm{T}}=v V$.

Proof. Both $M$ and $E_{+}$are closed and convex cones of $E$ with their vertices at the origin.

Since $M$ is a polyhedral (convex) cone in $E$ and $E_{+}$is a closed convex cone of $E$, then $E_{+}-M$ is a closed convex cone from Lemma of Clark [15], that is, $\overline{E_{+}-M}=E_{+}-M$. Thus $M-E_{+}$is a closed convex cone $\overline{M-E_{+}}=M-E_{+}$.

The frictionless market $(q, V)$ is strictly arbitrage-free if and only if $M \cap E_{+}=\{(0,0)\}$. We claim that $\left(E_{+} \backslash\{(0,0)\}\right) \cap\left(M-E_{+}\right)=\varnothing$. On the contrary, we assume that there exists $(r, t) \in\left(E_{+} \backslash\{(0,0)\}\right) \cap\left(M-E_{+}\right)$, then $(r, t) \in\left(E_{+} \backslash\{(0,0)\}\right)$ and $(r, t)=\left(r_{1}, t_{1}\right)-\left(r_{2}, t_{2}\right)$ for $\left(r_{1}, t_{1}\right) \in M$ and $\left(r_{2}, t_{2}\right) \in E_{+}$. Thus

$$
\left(r_{1}, t_{1}\right)=(r, t)+\left(r_{2}, t_{2}\right) \in M \cap\left(E_{+} \backslash\{(0,0)\}\right)
$$

contradicting the conclusion $M \cap E_{+}=\{(0,0)\}$ and $M \cap\left(E_{+} \backslash\{(0,0)\}\right)=\varnothing$.

Note that $M-\left(E_{+} \backslash\{(0,0)\}\right) \subseteq M-E_{+}$. It follows that $\overline{M-\left(E_{+} \backslash\{(0,0)\}\right)} \subseteq \overline{M-E_{+}}=M-E_{+}$. Thus $\left(E_{+} \backslash\{(0,0)\}\right) \cap \overline{M-\left(E_{+} \backslash\{(0,0)\}\right)}=\varnothing$. Since both $M$ and $E_{+} \backslash\{(0,0)\}$ are non-empty convex cones with vertices at the origin in the separable Banach space $E$, Theorem 5 in Clark [15] states that there exists a non-zero continuous linear functional $f: E \rightarrow \mathcal{R}$ on $E$ strictly separating $E_{+} \backslash\{(0,0)\}$ from $M$, that is, $f(n)>0$ for all $n \in E_{+} \backslash\{(0,0)\}$ and $f(m) \leq 0$ for all $m \in M$.

The condition $f(n)>0$ for all $n \in E_{+} \backslash\{(0,0)\}$ implies that $f: E \rightarrow \mathcal{R}$ is a strictly positive continuous linear functional on $E$. Thus $f$ is represented by some $\alpha \in \mathcal{R}_{++}$and $\beta \in T_{++}^{*}$ by $f(r, x)=\alpha r+\beta t$ for any $(r, t) \in E$.

Since $M$ is a linear space, $f(m)=0$ for all $m \in M$, that is, $-\alpha q^{\mathrm{T}} \theta+\beta V \theta=0$ for all $\theta \in \mathcal{R}^{J}$. It follows that $q^{\mathrm{T}}=\left(\frac{1}{\alpha} \beta\right) V$. The vector $\frac{1}{\alpha} \beta \in T_{++}^{*}$ is that we want.

The converse is again obvious. Q.E.D.

[Remark 1] If $\Omega$ is a finite set, then $T=\mathcal{R}^{\Omega}$ is an Euclidean space, Definition 1 is the usual concept of strict arbitrage-freeness. Theorem 2 is the well-known Stiemke's Lemma (Duffie [9,10]).

[Remark 2] Theorem 2 (The Extension of Stiemke's Lemma) means that the present value of the securities prices at date 0 is the value of their returns at date 1 .

[Remark 3] If $q^{\mathrm{T}}=v V$ where $v$ is a linear functional on $M=\operatorname{spanV}$, we can prove Theorem 2 by Kreps [18]. The sufficient condition is obvious, we only need prove the necessary condition. Take $M=\operatorname{spanV}$, then any element $m \in M$ is of the form $m=V \theta$ where $\theta \in \mathcal{R}^{J}$. Take $\pi$ as a linear functional on $M$ solving the equation $\pi(V \theta)=q^{\mathrm{T}} \theta$ for all $\theta \in \mathcal{R}^{J}$, i.e. $q^{\mathrm{T}}=\pi V$ and take $K=T_{+} \backslash\{0\}$. If the frictionless market $(q, V)$ is strictly arbitrage-free, then the pair $(M, \pi)$ admits no free lunches. Kreps' conditions (4.7) and (4.10) are applied, then the pair $(M, \pi)$ is $(T, \tau, K)$-viable from Theorem 3 (Kreps [18]), that is, the pair $(M, \pi)$ has the extension property for $(T, \tau, K)$. Thus the necessary condition is obtained.

\section{Frictional Security Markets}

Suppose that there are transaction costs in the trading, the coefficients $b^{j} \in[0, \infty)$ and $s^{j} \in[0,1)$ are respectively the transaction cost rates for purchasing and selling the security $j$. Then the algebraic cost induced by (buying) a position $\theta^{j} \geq 0$ units of security $j$ is $q^{j}\left(1+b^{j}\right) \theta^{j}$ and the algebraic gain induced by (selling) a position $\theta^{j} \leq 0$ units of security $j$ is $q^{j}\left(1-s^{j}\right) \theta^{j}$. We introduce the functions $\Phi^{j}: \mathcal{R} \rightarrow \mathcal{R}$ defined by 


$$
\Phi^{j}(z)= \begin{cases}q^{j}\left(1+b^{j}\right) z, & z \geq 0 \\ q^{j}\left(1-s^{j}\right) z, & z \leq 0\end{cases}
$$

and the functions $\phi^{j}: \mathcal{R} \rightarrow \mathcal{R}$ defined by

$$
\phi^{j}(z)= \begin{cases}\left(1+b^{j}\right) z, & z \geq 0 \\ \left(1-s^{j}\right) z, & z \leq 0 .\end{cases}
$$

Then $\Phi^{j}(z)=q^{j} \phi^{j}(z)$.

For any integer $N=1,2, \cdots$, a function $\psi: \mathcal{R}^{N} \rightarrow \mathcal{R}$ is subliner if, for any $x^{1} \in \mathcal{R}^{N}, \quad x^{2} \in \mathcal{R}^{N}, \quad x \in \mathcal{R}^{N}$ and $\lambda \in \mathcal{R}_{+}$,

$$
\psi\left(x^{1}+x^{2}\right) \leq \psi\left(x^{1}\right)+\psi\left(x^{2}\right) \text { and } \psi(\lambda x)=\lambda \psi(x) .
$$

The function $\phi^{j}$ is sublinear, and hence convex. Therefore the function $\Phi^{j}$ is also sublinear, and hence convex.

The total cost or gain induced by (trading) a portfolio $\theta \in \mathcal{R}^{J}$ is $\sum_{j=1}^{J} \Phi^{j}\left(\theta^{j}\right)=\sum_{j=1}^{J} q^{j} \phi^{j}\left(\theta^{j}\right)$. We define the function $\tau: \mathcal{R}^{J} \rightarrow \mathcal{R}$ by

$$
\tau(x)=\sum_{j=1}^{J} \Phi^{j}\left(x^{j}\right)=\sum_{j=1}^{J} q^{j} \phi^{j}\left(x^{j}\right) .
$$

Then the total cost or gain induced by (trading) a portfolio $\theta \in \mathcal{R}^{J}$ is $\tau(\theta)$. As we know, the function $\tau$ is sublinear, and hence convex.

[Definition 3] The frictional market $(q, V, b, s)$ is weakly arbitrage-free if any portfolio $\theta \in \mathcal{R}^{J}$ of securities has a positive total cost or gain $\tau(\theta) \geq 0$ whenever it has a positive payoff $V \theta \in T_{+}$.

We note that Definitions 2 and 2' are equivalent. However, in the presence of transaction costs, the corresponding Definitions 4 and 4' (as follows) are not. We establish Theorem 4 for Definition 4' of the strictly arbitrage-free frictional market $(q, V, b, s)$.

[Definition 4] The frictional market $(q, V, b, s)$ is strictly arbitrage-free if (1) any portfolio $\theta \in \mathcal{R}^{J}$ of securities has a strictly positive total cost or gain $\tau(\theta)>0$ whenever it has a positive non-zero payoff $V \theta \in T_{+} \backslash\{0\}$; and (2) any portfolio $\theta \in \mathcal{R}^{J}$ of securities has a zero total cost or gain $\tau(\theta)=0$ whenever it has a zero payoff $V \theta=0$.

[Definition 4'] The frictional market $(q, V, b, s)$ is strictly arbitrage-free if (1) any portfolio $\theta \in \mathcal{R}^{J}$ of securities has a strictly positive total cost or gain $\tau(\theta)>0$ whenever it has a positive non-zero payoff $V \theta \in T_{+} \backslash\{0\}$; and (2) any portfolio $\theta \in \mathcal{R}^{J}$ of securities has a positive total cost or gain $\tau(\theta) \geq 0$ whenever it has a positive payoff $V \theta \in T_{+}$.

Definition 4 obviously implies Definition 4'. Definition 4' does not imply Definition 4 because of the presence of friction. In the frictionless model, we define the marketed subspace

$$
M=\left\{\left(-q^{\mathrm{T}} \theta, V \theta\right) \in E \mid \theta \in \mathcal{R}^{J}\right\}
$$

of the space $E$ to prove the first fundamental theorems of asset pricing. In the frictional model, we can't consider the corresponding marketed "subspace" $\left\{(-\tau(\theta), V \theta) \in E \mid \theta \in \mathcal{R}^{J}\right\}$. In fact, this marketed "subspace" isn't a subspace of the space $E$. Instead, we define the subset $M^{\prime}$ in the space $E$ as follows

$$
M^{\prime}=\left\{(r, t) \in E \mid r \leq-\tau(\theta) \text { and } t=V \theta \text { for } \theta \in \mathcal{R}^{J}\right\}
$$

[Lemma 2] $M^{\prime}$ is a closed and convex cone in the space $E$.

Proof. $M^{\prime}$ is a closed cone obviously since the function $\tau$ is continuous and sublinear.

For any $\left(r^{1}, t^{1}\right) \in M^{\prime}$ and $\left(r^{2}, t^{2}\right) \in M^{\prime}$, there exist $\theta^{1} \in \mathcal{R}^{J}$ and $\theta^{2} \in \mathcal{R}^{J}$ such that $r^{1} \leq-\tau\left(\theta^{1}\right)$, 
$t^{1}=V \theta^{1}$ and $r^{2} \leq-\tau\left(\theta^{2}\right), t^{2}=V \theta^{2}$. Thus $r^{1}+r^{2} \leq-\tau\left(\theta^{1}\right)-\tau\left(\theta^{2}\right)=-\left[\tau\left(\theta^{1}\right)+\tau\left(\theta^{2}\right)\right] \leq-\tau\left(\theta^{1}+\theta^{2}\right)$ since the function $\tau$ is sublinear, and $t^{1}+t^{2}=V \theta^{1}+V \theta^{2}=V\left(\theta^{1}+\theta^{2}\right)$. Therefore $\left(r^{1}+r^{2}, t^{1}+t^{2}\right) \in M^{\prime}$. Since $M^{\prime}$ is a cone, $M^{\prime}$ is convex. Q.E.D.

For simplicity, we use the following notations in the subsequent sections.

$$
\mathbb{1}=\left(\begin{array}{c}
1 \\
\vdots \\
\vdots \\
1
\end{array}\right) \begin{aligned}
& 1 \\
& J
\end{aligned} \quad b=\left(\begin{array}{c}
b^{1} \\
\vdots \\
b^{J}
\end{array}\right) \text { and } s=\left(\begin{array}{c}
s^{1} \\
\vdots \\
s^{J}
\end{array}\right)
$$

We define the box product of two vectors $y_{1} \in \mathcal{R}^{N}$ and $y_{2} \in \mathcal{R}^{N}$ by

$$
y_{1} \square y_{2}=\left(\begin{array}{c}
y_{1}^{1} y_{2}^{1} \\
\vdots \\
y_{1}^{N} y_{2}^{N}
\end{array}\right)
$$

\section{Weakly Arbitrage-Free Security Valuation Theorem with Frictional Security Markets}

In this section, we assume that $T$ is a locally convex topological space.

[Proposition 3] The frictional market $(q, V, b, s)$ is weakly arbitrage-free if and only if $M^{\prime} \cap E_{+} \subseteq\{0\} \times\langle V\rangle_{+}$.

Proof. $(r, t) \in M^{\prime} \cap E_{+}$implies that there exists $\theta \in \mathcal{R}^{J}$ such that $0 \leq r \leq-\tau(\theta)$ and $t=V \theta \in T_{+}$. On the other hand, $V \theta \in T_{+}$implies that $\tau(\theta) \geq 0$ from Definition 3. Then $r=-\tau(\theta)=0$ and $t \in\langle V\rangle_{+}$, that is, $M^{\prime} \cap E_{+} \subseteq\{0\} \times\langle V\rangle_{+}^{+}$.

Conversely, if there exists $\theta \in \mathcal{R}^{J}$ such that $V \theta \in T_{+}$and $\tau(\theta)<0$, then $-\tau(\theta)>0$. Thus

$$
(-\tau(\theta), V \theta) \in M^{\prime} \cap E_{+} \text {and }(-\tau(\theta), V \theta) \notin\{0\} \times\langle V\rangle_{+} .
$$

This is a contradiction! Therefore, any portfolio $\theta \in \mathcal{R}^{J}$ of securities has a positive total cost or gain $\tau(\theta) \geq 0$ whenever it has a positive payoff $V \theta \in T_{+}$. Q.E.D.

[Remark] The proof of Proposition 3 only requires the property that $M^{\prime}$ is a closed and convex cone in the space $E$ and doesn't need the explicit definition of the function $\tau$, so does Proposition 3.

[Theorem 3] The frictional market $(q, V, b, s)$ is weakly arbitrage-free if and only if there exists a positive functional $v \in T_{+}^{*}$ satisfying

$$
q \square(11-s) \leq v V \leq q \square(11+b)
$$

Proof. Both $M^{\prime}$ and $E_{+}$are closed and convex cones of $E$ with their vertices at the origin.

The frictional market $(q, V, b, s)$ is weakly arbitrage-free if and only if $M^{\prime} \cap E_{+} \subseteq\{0\} \times\langle V\rangle_{+}$. Note that $M^{\prime} \cap E_{+}$is a convex set. Let $N^{\prime}=E_{+} \backslash M^{\prime}=E_{+} \backslash\left(M^{\prime} \cap E_{+}\right)$, then $N^{\prime}$ is a (non-empty) convex set. Both $M^{\prime}$ and $N^{\prime}$ are non-empty disjoint convex cones, $M^{\prime} \cap N^{\prime}=M^{\prime} \cap\left(E_{+} \backslash M^{\prime}\right)=\varnothing .(1,0) \notin \overline{M^{\prime}-N^{\prime}}$ implies $\overline{M^{\prime}-N^{\prime}} \neq E$. Clark [16] stated that there exists a non-zero continuous linear functional $f: E \rightarrow \mathcal{R}$ separating $N^{\prime}$ from $M^{\prime}$, that is, $f(n) \geq 0$ for all $n \in N^{\prime}$ and $f(m) \leq 0$ for all $m \in M^{\prime}$. Moreover, $f(1,0)>0$.

From the proof of Theorem $1, f: E \rightarrow \mathcal{R}$ is a positive continuous linear functional on $E$, and $f$ is represented by some $\alpha \in \mathcal{R}_{+}$and $\beta \in T_{+}^{*}$ by $f(r, t)=\alpha r+\beta t$ for any $(r, t) \in E$.

On the other hand, the fact that $f(m) \leq 0$ for all $m \in M^{\prime}$ implies $f(r, t)=\alpha r+\beta t \leq 0$ for all $r \leq-\tau(\theta)$ and $t=V \theta$ for $\theta \in \mathcal{R}^{J}$. Then $-\alpha \tau(\theta)+\beta V \theta \leq 0$ for all $\theta \in \mathcal{R}^{J}$. And hence we have

$$
-\alpha \sum_{j=1}^{J} q^{j} \phi^{j}\left(\theta^{j}\right)+\beta \sum_{j=1}^{J} V^{j} \theta^{j} \leq 0
$$

for all $\theta \in \mathcal{R}^{J}$. For each $j=1, \cdots, J$, assigning $\theta^{j^{\prime}}=\left\{\begin{array}{ll}-1, & j^{\prime}=j \\ 0, & j^{\prime} \neq j\end{array}\right.$, we have

$$
-\alpha q^{j} \phi^{j}(-1)-\beta V^{j} \leq 0
$$


that is, $\alpha q^{j}\left(1-s^{j}\right)-\beta V^{j} \leq 0$. Thus

$$
q^{j}\left(1-s^{j}\right) \leq\left(\frac{1}{\alpha} \beta\right) V^{j} .
$$

For each $j=1, \cdots, J$, we assign $\theta^{j^{\prime}}=\left\{\begin{array}{ll}1, & j^{\prime}=j \\ 0, & j^{\prime} \neq j\end{array}\right.$ instead, it follows that

$$
-\alpha q^{j} \phi^{j}(1)+\beta V^{j} \leq 0 .
$$

that is, $-\alpha q^{j}\left(1+b^{j}\right)+\beta V^{j} \leq 0$. Thus

$$
\left(\frac{1}{\alpha} \beta\right) V^{j} \leq q^{j}\left(1+b^{j}\right)
$$

Therefore, for any $j=1, \cdots, J$,

$$
q^{j}\left(1-s^{j}\right) \leq\left(\frac{1}{\alpha} \beta\right) V^{j} \leq q^{j}\left(1+b^{j}\right)
$$

The vector $v=\frac{1}{\alpha} \beta \in T_{+}^{*}$ is thus what we want:

$$
q \square(11-s) \leq v V \leq q \square(11+b) .
$$

Conversely, assume that there exists a positive functional $v \in T_{+}^{*}$ satisfying

$$
q \square(11-s) \leq v V \leq q \square(11+b) .
$$

For any $j=1, \cdots, J$,

$$
q^{j}\left(1-s^{j}\right) \leq v V^{j} \leq q^{j}\left(1+b^{j}\right)
$$

For any $\theta^{j} \leq 0$,

$$
v V^{j} \theta^{j} \leq q^{j}\left(1-s^{j}\right) \theta^{j}
$$

And for any $\theta^{j} \geq 0$,

$$
v V^{j} \theta^{j} \leq q^{j}\left(1+b^{j}\right) \theta^{j}
$$

Thus

$$
v V^{j} \theta^{j} \leq q^{j} \phi^{j}\left(\theta^{j}\right)=\Phi^{j}\left(\theta^{j}\right)
$$

Summarying over $j=1, \cdots, J$, we then have, for any $\theta \in \mathcal{R}^{J}$,

$$
v V \theta=\sum_{j=1}^{J} v V^{j} \theta^{j} \leq \sum_{j=1}^{J} \Phi^{j}\left(\theta^{j}\right)=\tau(\theta)
$$

Therefore the frictional market $(q, V, b, s)$ is weakly arbitrage-free. Q.E.D.

[Remark] Theorem 3 means the first fundamental theorem of weakly arbitrage-freeness with frictional security markets. If there exists no transaction costs, that is, $b=0$ and $s=0$, then Theorem 3 reduces to Theorem 1 .

\section{Strictly Arbitrage-Free Security Valuation Theorem with Frictional Security Markets}

In this section, we assume that $T$ is a separable Banach space. We prove the following Proposition 4 and Theorem 4 for Definition 4' of the strictly arbitrage-free frictional market $(q, V, b, s)$.

[Proposition 4] The frictional market $(q, V, b, s)$ is strictly arbitrage-free if and only if $M^{\prime}$ and $E_{+}$ intersect precisely at $(0,0)$, that is, $M^{\prime} \cap E_{+}=\{(0,0)\}$. 
Proof. $(r, t) \in M^{\prime} \cap E_{+}$implies that there exists $\theta \in \mathcal{R}^{J}$ such that $0 \leq r \leq-\tau(\theta)$ and $t=V \theta \in T_{+}$. If $V \theta \in T_{+} \backslash\{0\}$, then $\tau(\theta)>0$ from Definition $4^{\prime}(1), 0 \leq r \leq-\tau(\theta)<0$, which is a contradiction! If $V \theta=0 \in T_{+}$, then $\tau(\theta) \geq 0$ from Definition $4^{\prime}(2), r=-\tau(\theta)=0$ and $t=V \theta=0 \in T_{+}$. Thus $M^{\prime} \cap E_{+} \subseteq\{(0,0)\}$, and hence $M^{\prime} \cap E_{+}=\{(0,0)\}$.

For sufficiency, we must consider two cases: (1) If there exists $\theta \in R^{J}$ such that $V \theta \in T_{+} \backslash\{0\}$ and $\tau(\theta) \leq 0$ then $-\tau(\theta) \geq 0$. Take $(r, t) \in M^{\prime} \cap E_{+}$such that $0 \leq r \leq-\tau(\theta)$ and $t=V \theta$, then $(0,0) \neq(r, t) \in M^{\prime} \cap E_{+}$, which is a contradiction. It follows that any portfolio $\theta \in \mathcal{R}^{J}$ of securities has a strictly positive total cost or gain $\tau(\theta)>0$ whenever it has a positive payoff $V \theta \in T_{+} \backslash\{0\}$. (2) If there exists $\theta \in \mathcal{R}^{J}$ such that $V \theta \in T_{+}$ and $\tau(\theta)<0$, then $-\tau(\theta)>0$. Take $(r, t) \in M^{\prime} \cap E_{+}$such that $0<r \leq-\tau(\theta)$ and $t=V \theta$, then

$(0,0) \neq(r, t) \in M^{\prime} \cap E_{+}$, which is a contradiction! The two contradictions mean that the frictional market $(q, V, b, s)$ is strictly arbitrage-free. Q.E.D.

[Remark] The proof of Proposition 4 doesn't use the representation of the function $\tau$, then Proposition 4 holds for usual total cost or gain function.

[Theorem 4] The The frictional market $(q, V, b, s)$ is strictly arbitrage-free if and only if there exists a strictly positive functional $v \in T_{++}^{*}$ satisfying

$$
q \square(11-s) \leq v V \leq q \square(11+b)
$$

Proof. Both $M^{\prime}$ and $E_{+}$are closed and convex cones of $E$ with the vertices at the origin.

Since $M^{\prime}$ is a polyhedral (convex) cone in $E$ and $E_{+}$is a closed convex cone of $E, E_{+}-M^{\prime}$ is a closed convex cone from Lemma of Clark [15], that is, $\overline{E_{+}-M^{\prime}}=E_{+}-M^{\prime}$. Thus $M^{\prime}-E_{+}$is a closed convex cone $\overline{M^{\prime}-E_{+}}=M^{\prime}-E_{+}$.

The frictional market $(q, V, b, s)$ is strictly arbitrage-free if and only if $M^{\prime} \cap E_{+}=\{(0,0)\}$. We claim that

$$
\left(E_{+} \backslash\{(0,0)\}\right) \cap\left(M^{\prime}-E_{+}\right)=\varnothing .
$$

On the contrary, we assume that there exists $(r, t) \in\left(E_{+} \backslash\{(0,0)\}\right) \cap\left(M^{\prime}-E_{+}\right)$. Then $(r, t) \in\left(E_{+} \backslash\{(0,0)\}\right)$ and $(r, t)=\left(r_{1}, t_{1}\right)-\left(r_{2}, t_{2}\right)$ for $\left(r_{1}, t_{1}\right) \in M^{\prime}$ and $\left(r_{2}, t_{2}\right) \in E_{+}$. Thus

$$
\left(r_{1}, t_{1}\right)=(r, t)+\left(r_{2}, t_{2}\right) \in M^{\prime} \cap\left(E_{+} \backslash\{(0,0)\}\right),
$$

contradicting the fact that $M^{\prime} \cap\left(E_{+} \backslash\{(0,0)\}\right)=\varnothing \quad$ (which follows from $M^{\prime} \cap E_{+}=\{(0,0)\}$ in Proposition 4).

Note that $M^{\prime}-\left(E_{+} \backslash\{(0,0)\}\right) \subseteq M^{\prime}-E_{+}$, then

$$
\overline{M^{\prime}-\left(E_{+} \backslash\{(0,0)\}\right)} \subseteq \overline{M^{\prime}-E_{+}}=M^{\prime}-E_{+} .
$$

Thus, $\left(E_{+} \backslash\{(0,0)\}\right) \cap \overline{M^{\prime}-\left(E_{+} \backslash\{(0,0)\}\right)}=\varnothing$. Since both $M^{\prime}$ and $E_{+} \backslash\{(0,0)\}$ are non-empty convex cones with vertices at the origin in the separable Banach space $E$, Theorem 5 in Clark [15] states that there exists a non-zero continuous linear functional $f: E \rightarrow \mathcal{R}$ on $E$ strictly separating $E_{+} \backslash\{(0,0)\}$ from $M^{\prime}$. That is, $f(n)>0$ for all $n \in E_{+} \backslash\{(0,0)\}$ and $f(m) \leq 0$ for all $m \in M^{\prime}$.

The condition $f(n)>0$ for all $n \in E_{+} \backslash\{(0,0)\}$ implies that $f: E \rightarrow \mathcal{R}$ is a strictly positive continuous linear functional on $E$. Thus $f$ is represented by some $\alpha \in \mathcal{R}_{++}$and $\beta \in T_{++}^{*}$ by $f(r, x)=\alpha r+\beta t$ for any $(r, t) \in E$.

The next proof is the same as the corresponding part in Theorem 3. Q.E.D.

[Remark] Theorem 4 means the first fundamental theorem of strict arbitrage-freeness with frictional security markets. If there exists no transaction costs, that is, $b=0$ and $s=0$, then Theorem 4 reduces to Theorem 2.

\section{Concluding Remarks}

In Section 4, we examine the following problem: whether the security prices $q \in \mathcal{R}^{J}$ can be represented by strictly positive continuous linear functional $v \in T^{*}$ as $q^{T}=v V$. More precisely, for the security prices 
$q \in \mathcal{R}^{J}$ and the returns $V^{j} \in T, j=1, \cdots, J$, we study the existence of strictly positive continuous linear functional $v \in T^{*}$ as $q^{\mathrm{T}}=v V$. Ross [29] and Kreps [18] studied how to extend a linear functional $v$ on $M=\operatorname{span} V$ to $T$ that is strictly positive continuous. However, the existence of the linear functional $v$ on $M$ with $q^{\mathrm{T}}=v V$ is not at all an assured condition. It holds under certain conditions. For example, if $q^{\mathrm{T}}=v V$, our result is an analogue of theirs. In the equation $q^{\mathrm{T}}=v V$, each linear functional $v$ on $M=s p a n V$ corresponds to a vector $q \in \mathcal{R}^{J}$ and each vector $q \in \mathcal{R}^{J}$ corresponds to a linear functional $v$ on $M$ only if $V$ is column linear independent. Therefore, our results in Section 4 are the same as theirs under the assumption " $V$ is column linear independent". But in general, this may not hold.

Dalang, Morton and Willinger [20] and Jacod and Sgiryaev [22] studied arbitrage-free model, weakly arbitrage-free model, and strongly arbitrage-free model, provided simple proofs of the two fundamental theorems of asset pricing theory. Jacod and Sgiryaev [22] proved that the three concepts are equivalent to each another. In fact, the three concepts are strictly arbitrage-free securities' price-return pair in Section 2 of our paper. Jacod and Sgiryaev [22] assumed that $V$ is an integrable (finite expectation) random variable (martingale condition). But we only assume $V$ is in the locally convex topological space $T$ for weakly arbitragefree frictionless market (Section 3), and $V$ is in the separable Banach space for strictly arbitrage-free frictionless market (Section 4). Therefore our study in Sections 2, 3 and 4 is more general than Jacod and Sgiryaev [22].

Pham and Touzi [26] studied frictional markets with the transaction cost rates for purchasing and selling the securities, addressed the problem of characterization of no arbitrage opportunity (strict arbitrage-freeness) in the presence of transaction costs in a discrete-time financial model, and extended the fundamental theorem of asset pricing under a non-degeneracy assumption. We also study the first fundamental valuation theorems of asset pricing from frictionless security markets to frictional security markets in Sections 5, 6 and 7. However, our results are different from those in Pham and Touzi [26] in the following two aspects. (1) The definition of no arbitrage opportunity in Pham and Touzi [26] is different from our definition of strict arbitrage-freeness. (2) Our proofs of Theorems 3 and 4 are different from Pham and Touzi [26]. In fact, we provide the proof by using theory of functional analysis, while Pham and Touzi [26] applied stochastic analysis to prove their results. Therefore, our result works for more general space than theirs.

Frictional economies are fundamentally different from their frictionless counterparts. The theory of general economic equilibrium for frictional economies with incomplete financial markets should be studied. We make the first step by establishing the corresponding no-arbitrage (that is, strictly arbitrage-free) pricing theory. From the first fundamental valuation theorems of asset pricing in general state space with transaction costs we have obtained here, one may further study the corresponding existence of general equilibrium for frictional economy with infinite-dimensional commodity space and incomplete financial markets.

In Section 7, we proved the equivalent conditions (Proposition 4 and Theorem 4) of strictly arbitrage-free frictional market by using Definition 4'. There are some difference between Definitions 4 and 4'. In fact, we can obtain the following (weaker) results for strictly arbitrage-free frictional market as defined by Definition 4.

[Proposition] If the frictional market $(q, V, b, s)$ is strictly arbitrage-free, then $M^{\prime}$ and $E_{+}$intersect precisely at $(0,0)$, that is, $M^{\prime} \cap E_{+}=\{(0,0)\}$.

[Theorem] If the The frictional market $(q, V, b, s)$ is strictly arbitrage-free, then there exists a strictly positive functional $v \in T_{++}^{*}$ satisfying

$$
q \square(11-s) \leq v V \leq q \square(11+b)
$$

The proofs of Proposition and Theorem are from the proofs of Proposition 4 and Theorem 4. Conversely, we can't check the sufficiency. The proof of Theorem 4 holds, too. If

$$
q \square(11-s) \leq v V \leq q \square(11+b)
$$

where $v \in T_{++}^{*}$ is a strictly positive vector, then, for any $\theta \in \mathcal{R}^{J}$,

$$
v V \theta=\sum_{j=1}^{J} v V^{j} \theta^{j} \leq \sum_{j=1}^{J} \Phi^{j}\left(\theta^{j}\right)=\tau(\theta)
$$

then

$$
v V \theta \leq \tau(\theta) \text { and } \quad v V(-\theta) \leq \tau(-\theta)
$$


that is,

$$
-\tau(-\theta) \leq v V \theta \leq \tau(\theta)
$$

If any portfolio $\theta \in \mathcal{R}^{J}$ of securities has a zero payoff $V \theta=0$, then it has a positive total cost or gain $\tau(\theta) \geq 0$ for the portfolio $\theta \in \mathcal{R}^{J}$, and a positive total cost or gain $\tau(-\theta) \geq 0$ for the portfolio $-\theta \in \mathcal{R}^{J}$, thus

$$
-\tau(-\theta) \leq 0 \leq \tau(\theta)
$$

For any portfolio $\theta \in \mathcal{R}^{J}$ of securities, we can't obtain a zero total cost or gain $\tau(\theta)=0$ when it has a zero payoff $V \theta=0$. That is to say, we can't make sure that Definition 4 (2) holds. We can't come to the conclusion that the frictional market $(q, V, b, s)$ is strictly arbitrage-free in the sense of Definition 4.

\section{Funding}

This work is supported by Social Sciences and Humanities Research Council of Canada (SSHRC Insight Development Grant Number: 430-2012-0698), National Natural Science Foundation of China (NSFC Grant Numbers: 70825003 and 71273271) Major Basic Research Plan of Renmin University of China (Grant Number: 14XNL001)

\section{REFERENCES}

[1] D. Duffie and W. Shafer, “Equilibrium in Incomplete Markets I: A Basic Model of Generic Existence,” Journal of Mathematical Economics, Vol. 14, No. 3, 1985, pp. 285-300. http://dx.doi.org/10.1016/0304-4068(85)90004-7

[2] D. Duffie and W. Shafer, “Equilibrium in Incomplete Markets II: Generic Existence in Stochastic Economies,” Journal of Mathematical Economics, Vol. 15, No. 3, 1986, pp. 199-216. http://dx.doi.org/10.1016/0304-4068(86)90010-8

[3] J. Geanakoplos, “An Introduction to General Equilibrium with Incomplete Asset Markets,” Journal of Mathematical Economics, Vol. 19, No. 1-2, 1990, pp. 1-38. http://dx.doi.org/10.1016/0304-4068(90)90034-7

[4] J. Geanakoplos and W. Shafer, "Solving Systems of Simultaneous Equations in Economics,” Journal of Mathematical Economics, Vol. 19, No. 1-2, 1990, pp. 69-93. http://dx.doi.org/10.1016/0304-4068(90)90036-9

[5] M. D. Hirsch, M. J. P. Magill and A. Mas-Colell, “A Geometric Approach to a Class of Equilibrium Existence Theorems,” Journal of Mathematical Economics, Vol. 19, No. 1-2, 1990, pp. 95-106. http://dx.doi.org/10.1016/0304-4068(90)90037-A

[6] S. Y. Husseini, J.-M. Lasry and M. J. P. Magill, "Existence of Equilibrium with Incomplete Asset Markets,” Journal of Mathematical Economics, Vol. 19, No. 1-2, 1990, pp. 39-67. http://dx.doi.org/10.1016/0304-4068(90)90035-8

[7] M. Magill and W. Shafer, "Incomplete Markets,” In: W. Hildenbrand and H. Sonnenschein, Eds., Handbook of Mathematical Economics (Volume 4), Elsevier Science, North Holland, Amsterdam, 1991, pp. 1523-1614.

[8] D. Duffie, “Stochastic Equilibria with Incomplete Financial Markets,” Journal of Economic Theory, Vol. 41, No. 2, 1987, pp. 405-416. http://dx.doi.org/10.1016/0022-0531(87)90027-5

[9] D. Duffie, “Security Markets: Stochastic Models,” Stanford University, Academic Press, Stanford, 1988.

[10] D. Duffie, “Dynamic Asset Pricing Theory,” Princeton University, Academic Press, Princeton, 1996.

[11] M. Florenzano and P. Gourdel, “T-period Economies with Incomplete Markets,” Economics Letter, Vol. 44, No. 1-2, 1994, pp. 91-97. http://dx.doi.org/10.1016/0165-1765(93)00308-B

[12] J. Werner, "Equilibrium of Economies with Incompleete Financial Markets," Journal of Economic Theory, Vol. 36, No. 1, 1985, pp. 110-119. http://dx.doi.org/10.1016/0022-0531(85)90081-X

[13] J. Werner, "Structure of Financial Markets and Real Indeterminacy of Equilibrium,” Journal of Mathematical Economics, Vol. 19, No. 1-2, 1990, pp. 217-232. http://dx.doi.org/10.1016/0304-4068(90)90043-9

[14] S. Zhang, "Existence of Stochastic Equilibrium with Incomplete Financial Markets,” Applied Mathematics_Journal of Chinese Universities, Vol. 13, No. 1, 1998, pp. 77-94.

[15] S. A. Clark, “The Valuation Problem in Arbitrage Pricing Theory,” Journal of Mathematical Economics, Vol. 22, No. 5, 1993, pp. 463-478. http://dx.doi.org/10.1016/0304-4068(93)90037-L

[16] S. A. Clark, “Vector Space Methods in Additive Theory,” Journal of Mathematical Economics, conditionally accepted, 1994.

[17] J. M. Harrison and D. M. Kreps, “Martingales and Arbitrage in Multiperiod Securities Markets,” Journal of Economic Theory, Vol. 20, No. 3, 1979, pp. 381-408. http://dx.doi.org/10.1016/0022-0531(79)90043-7

[18] D. M. Kreps, “Arbitrage and Equilibrium in Economies with Infinitely Many Commodities,” Journal of Mathematical Eco- 
nomics, Vol. 8, No. 1, 1981, pp. 15-35. http://dx.doi.org/10.1016/0304-4068(81)90010-0

[19] J. M. Harrison and S. R. Pliska, "Martingales and Stochastic Integrals in the Theory of Continuous Trading," Stochastic Processes and Their Applications, Vol. 11, No. 3, 1981, pp. 215-260. http://dx.doi.org/10.1016/0304-4149(81)90026-0

[20] R. C. Dalang, A. Morton and W. Willinger, "Equivalent Martingale Measures and No-Arbitrage in Stochastic Securities Market Models,” Stochastics and Stochastic Reports, Vol. 29, No. 2, 1990, pp. 185-201. http://dx.doi.org/10.1080/17442509008833613

[21] K. Back and S. R. Pliska, “On the Fundamental Theorem of Asset Pricing with an Infinite State Space,” Journal of Mathematical Economics, Vol. 20, No. 1, 1991, pp. 1-18. http://dx.doi.org/10.1016/0304-4068(91)90014-K

[22] J. Jacod and A. N. Sgiryaev, "Local Martingales and the Fundamental Asset Pricing Theorems in the Discrete-Time Case," Finance and Stochastics, Vol. 2, No. 3, 1998, pp. 259-273. http://dx.doi.org/10.1007/s007800050040

[23] Z. Chen, “Financial Innovation and Arbitrage Pricing in Frictional Economics,” Journal of Economic Theory, Vol. 65, No. 1, 1995, pp. 117-135. http://dx.doi.org/10.1006/jeth.1995.1004

[24] E. Jouini and H. Kallal, "Martingales and Arbitrage in Securities Markets with Transaction Costs," Journal of Economic Theory, Vol. 66, No. 1, 1995, pp. 178-197. http://dx.doi.org/10.1006/jeth.1995.1037

[25] E. Jouini and H. Kallal, “Arbitrage in Securities Markets with Short-Sales Constraints,” Mathematical Finance, Vol. 5, No. 3, 1995, pp. 197-232. http://dx.doi.org/10.1111/j.1467-9965.1995.tb00065.x

[26] H. Pham and N. Touzi, "The Fundamental Theorem of Asset Pricing with Cone Constraints," Journal of Mathematical Economics, Vol. 31, No. 2, 1999, pp. 265-279. http://dx.doi.org/10.1016/S0304-4068(97)00059-1

[27] J. Farkas, “Uber die Theorie der Einfachen Ungleichungen,” Journal für die Reine und Angewandte Mathematik, Vol. 1902, No. 124, 1902, pp. 1-24.

[28] J. Franklin, “Methods of Mathematical Economics,” Springer-Verlag, New York, 1980.

[29] S. A. Ross, “A Simple Approach to the Valuation of Risky Streams,” Journal of Business, Vol. 51, No. 3, 1978 , pp. $453-475$. http://dx.doi.org/10.1086/296008 